\title{
Conflitos conjugais e familiares e presença de transtorno de déficit de atenção e hiperatividade (TDAH) na prole: revisão sistemática
}

\author{
Marital and familial conflicts and attention-deficit hyperactivity \\ disorder (ADHD) in the offspring: systematic review
}

Priscilla Rodrigues Guilherme', Paulo Mattos', Maria Antonia Serra-Pinheiro', Maria Angélica Regalla'

\section{RESUMO}

O transtorno do déficit de atenção e hiperatividade (TDAH) é um dos transtornos neurobiológicos com maior prevalência na infância e pode implicar dificuldades no funcionamento conjugal dos pais das crianças afetadas, bem como sofrer influência do mesmo. Objetivo: Realizar uma revisão sistemática acerca dos aspectos conjugais em famílias de crianças com TDAH, relacionando-os com outras variáveis, como a presença de comorbidades, aspectos socioeconômicos e saúde mental dos pais. Métodos: Revisão sistemática de literatura por meio do PubMed entre os anos de 1996 e 2006, utilizando os termos "ADD", "ADHD", "Attention-Deficit Hyperactivity Disorder", "Attention-Deficit", "marital conflict" e "family". Resultados: Dezesseis estudos dentre 628 publicações iniciais e 55 artigos posteriormente incluídos pelas referências bibliográficas foram avaliados. O relacionamento conjugal aparece comprometido em grande parte da literatura, principalmente nos pais daquelas crianças que apresentam distúrbio desafiador e de oposição (DDO) ou distúrbio de conduta comórbidos. Contudo, resultados opostos também são encontrados em diversas pesquisas.

\section{Palavras-chave}

Transtorno de déficit de atenção/hiperatividade, funcionamento conjugal, família.
Conclusões: Os resultados de pesquisas referentes ao funcionamento conjugal de pais destas crianças são heterogêneos. É preciso que sejam feitos estudos longitudinais, que possam esclarecer o impacto do transtorno nos conflitos conjugais de pais de portadores, bem como a influência destes últimos na expressão clínica do transtorno.

\begin{abstract}
Attention-deficit/hyperactivity disorder (ADHD) is one of the most common neurobiological disorders among children and might either influence or be influenced by problems in marital functioning of the parents of affected children. Studies aimed at investigating marital functioning in parents of these children have found controversial results. Objective: To perform a systematic review about the marital aspects of families with ADHD children and, to correlate those aspects with other variables, like the presence of comorbidities, socioeconomical aspects and parents' mental health. Methods: A systematic review of the literature was conducted in Pubmed between 1996 and 2006, using the following keywords: "ADD", "ADHD", "Attention-Deficit Hyperactivity
\end{abstract}

1 Grupo de Estudos de Déficit de Atenção do Instituto de Psiquiatria da Universidade Federal do Rio de Janeiro (Geda-IPUB/UFRJ).

Endereço para correspondência: Priscilla Guilherme

Av. das Américas, 500, bloco 20, sala 213 - Barra da Tijuca - 22640-100, Rio de Janeiro, RJ

E-mail: priscilla.psi@gmail.com 


\section{Key-words}

Attention-deficit/

hyperactivity disorder, marital functioning, family.
Disorder", "Attention-Deficit", "marital conflict", and "family". Results: Sixteen of the 628 initial articles and 55 additional papers included in a following phase, based on bibliographical references, were evaluated. Impairment in marital relationships has been documented in most studies, specially when ADHD is comorbid with Oppositional-Defiant Disorder (ODD) or Conduct Disorder (CD). However, studies indicating normal marital functioning also exist. Conclusions: Study results concerning ADHD and marital dysfunction have conflicting results. Futures longitudinal studies will be necessary to clarify the impact of ADHD in marital conflict of parents of children with $A D H D$, and also to determine the influence of the marital conflicts in the clinical expression of the disorder.

\section{INTRODUÇÃO}

O transtorno de déficit de atenção e hiperatividade (TDAH) é um transtorno do desenvolvimento, de forte influência neurobiológica, que atinge cerca de 4\% a 6\% das crianças em idade escolar (Rohde et al., 2004; Vasconcelos et al., 2005), sendo um dos distúrbios mais comuns na infância e uma das principais causas de procura de atendimento em ambulatórios de saúde mental. O TDAH é caracterizado por um padrão persistente de desatenção e/ou hiperatividade e impulsividade, que devem se manifestar em, no mínimo, dois ambientes (casa e escola, por exemplo) (American Psychiatric Association, 2000). Alguns destes sintomas devem estar presentes desde antes dos 7 anos de idade, além de estarem associados a comprometimento em uma variedade de contextos na vida da criança, podendo ainda levar a significativas dificuldades acadêmicas, sociais, profissionais e interpessoais em casos de persistência na vida adulta (Barkley, 2002).

Além do comprometimento nos diversos ambientes anteriormente mencionados, os prejuízos causados pelo TDAH podem também atingir o funcionamento familiar (Hechtman, 1996) e o relacionamento conjugal dos pais do portador (Harpin, 2005). Há estudos realizados em diversos países com pais de crianças portadoras do transtorno demonstrando maior prevalência de insatisfação e conflito conjugal, presença de sintomas psíquicos (como ansiedade e depressão) e alterações nas relações pai-filho como estilos disciplinares altamente diretivos e hostis ou excessivamente permissivos (Johnston e Mash, 2001).

Ao longo dos últimos 10 anos, tem ocorrido um avanço significativo no que diz respeito à compreensão de fatores ligados à natureza biológica do TDAH (Faraone e Biederman, 1998; Berger e Posner, 2000). Entretanto, os estudos que investigaram as variáveis sociais e interpessoais parecem ser descritos de forma menos sistemática e sem atualização continuada (Johnston e Mash, 2001).

Embora fatores familiares não sejam apontados como causa do transtorno, diversas pesquisas indicam a importância do contexto familiar no TDAH. A disfunção familiar pode constituir fator de risco que, ao interagir com a predisposição neurobiológica da criança, exacerba a expressão dos sintomas e modifica o curso do transtorno (Carlson et al., 1995; Hechtman, 1996; Miranda et al., 2002; Presentación et al., 2006).

Este artigo tem como objetivo realizar uma revisão sistemática da literatura sobre o funcionamento familiar e o relacionamento conjugal nos pais de crianças com TDAH, considerando os diversos fatores associados e potencialmente confundidores, como nível socioeconômico, tipo de TDAH e presença de comorbidades, por exemplo, o distúrbio desafiador e de oposição.

\section{MÉTODO}

Foram analisados todos os artigos em língua inglesa e portuguesa com os termos "ADD", "ADHD", "Attention-Deficit Hyperactivity Disorder", "Attention-Deficit", "marital conflict" e "family" na base de dados PubMed, de 1996 a 2006. Foram encontrados 628 resultados, dos quais 16 artigos foram incluídos, dentre estes três revisões. A maioria dos artigos apresentados na busca não era dirigido ao tema $e$ foi excluída. Artigos que não versavam especificamente sobre o tema referido, como os que eram dirigidos ao uso de medicação e a outros transtornos que não o TDAH, assim como relatos de caso não foram incluídos. Além dos artigos encontrados na busca, também foram incluídas outras 55 publicações, referenciadas nos artigos, que foram consideradas importantes para o entendimento do presente tema.

\section{O funcionamento familiar e o desenvolvimento infantil}

O entendimento dos diversos problemas de comportamento infantil requer consideração, não apenas sobre fatores individuais (sejam eles neurobiológicos ou psicológicos), mas também a compreensão do ambiente em que a criança se desenvolve e como este pode contribuir para a manutenção e/ou agravamento dos quadros clínicos, incluindo aí o estudo de aspectos familiares e parentais (Lindahl, 1998). 
Nesta perspectiva, uma boa interação com o adulto parece ser elemento essencial, contribuindo para que a criança desenvolva uma melhor percepção das pessoas, objetos e símbolos, modulando seu comportamento e adquirindo os conhecimentos e as habilidades necessários ao desenvolvimento (Bronfenbrenner e Ceci, 1994). A família exerce influência importante na socialização da criança, que, por sua vez, se constitui fator de relevância para o desenvolvimento cognitivo. O núcleo familiar realiza o papel de mediação entre a criança e a sociedade, sendo, portanto, o meio básico pelo qual a criança começa a estabelecer suas relações com o mundo (Souza, 1997).

Diversos aspectos familiares possuem impacto significativo sobre o desenvolvimento cognitivo infantil, como a escolaridade materna (Bradley e Corwyn, 2002), a estimulação ambiental (Andrade et al., 2005) e o nível socioeconômico (Zamberlan e Biasoli-Alves apud Andrade et al., 2005). Cumpre ressaltar que a estimulação recebida pela criança associa-se positivamente ao nível de escolaridade materna, o que demonstra a importância dos papéis da dinâmica familiar e das condições materiais para o desenvolvimento cognitivo infantil (Andrade et al., 2005).

Nos últimos anos, o relacionamento conjugal tem sido apontado como fator de grande importância para a qualidade de vida das famílias, principalmente no que diz respeito à qualidade do relacionamento que pais e mães estabelecem com seus filhos (Belsky, 1984; Gottman, 1998).

Grych et al. (1992) sugeriram que a presença de conflitos hostis e agressivos estaria mais relacionada a problemas externalizantes (como o TDAH), enquanto os problemas internalizantes (como a depressão e a ansiedade) resultariam das avaliações das crianças sobre eles, ou seja, da maneira como as crianças interpretavam o conflito. O conflito marital pode ser entendido como um estressor que, quando combinado a outros aspectos da vida da criança, pode se associar a problemas de ajustamento (Fincham et al., 1994). Belsky (1984) propôs ainda que relacionamentos conjugais e relação pais-filho seriam fatores interdependentes, e que os conflitos ocorridos no casamento não afetariam apenas a maneira como os pais tratam os seus filhos, mas também a forma como as crianças lidam com os seus pais.

Embora alguns estudos demonstrem uma associação significativa entre as dificuldades no relacionamento marital e o desenvolvimento das crianças, em geral, o modo como estas variáveis se relacionam permanecem sem explicação satisfatória. Interações conjugais conflituosas podem influenciar a criança tanto diretamente, por meio de um processo de modelação (aprendizagem de comportamentos pela observação de modelos), quanto indiretamente, pelas práticas parentais inconsistentes e discordantes (Emery, 1992 apud Johnston; Mash, 2001; Fincham et al., 1994).

Segundo Braz et al. (2005), casamentos com relações insatisfatórias se associam à maior insensibilidade dos ge- nitores e a desenvolvimento infantil pouco ajustado. Erel e Burman (1995) também sugeriram que casais que mantêm um relacionamento insatisfatório e conflituoso se tornam mais desequilibrados emocionalmente e irritáveis, influenciando seus comportamentos como cuidadores, sendo menos sensíveis e atenciosos com suas crianças.

Belsky (1984) propôs que o relacionamento marital funcionaria como um suporte primário para a paternidade. Dessa forma, a discordância freqüente no casamento poderia levar a dificuldades no estabelecimento de práticas parentais efetivas. Em concordância com esta proposta, alguns autores (Fauber e Long, 1991) sugeriram que o conflito marital só afetaria o desenvolvimento das crianças em virtude de suas conseqüências diretas nas práticas parentais. De acordo com a proposta de Fincham et al. (1994), a tensão no relacionamento marital pode levar à inconsistência nos comportamentos disciplinares utilizados pelos pais. Estas diferenças, por sua vez, podem ser expressas abertamente e acarretar insatisfação entre o casal e uma insatisfação mais saliente com a criança. Em alguns casos, um dos cônjuges pode passar a defender a criança e se opor ao seu parceiro, resultando aumento do conflito marital. Este cônjuge pode se frustrar, e passar a utilizar táticas autoritárias (incluindo agressões físicas e/ou verbais), tornando-se cada vez menos envolvido na função parental.

Wolfe (1985) sugere ainda que quando os pais são agressivos um com o outro, há uma tendência maior de que a agressão também se dirija para a criança. Apesar da existência de diferenças individuais nas respostas cognitivas e emocionais das crianças a essas relações conjugais, certos tipos de conflitos entre o casal parecem eliciar modos parecidos de se comportar, como as agressões verbais, a hostilidade e as auto-acusações (Grych e Fincham, 1993).

Fincham et al. (1994) sugeriram que embora a exposição ao conflito marital fosse um fator estressor para a criança, este, isoladamente, não seria suficiente para explicar o seu efeito. Os autores sugeriram que a avaliação feita pela criança sobre o conflito e seu impacto poderia explicar meIhor aquela relação. Assim, a percepção da criança seria um mediador dos efeitos do conflito, o que também explicaria o porquê de as crianças reagirem diferentemente quando expostas a interações maritais disfuncionais.

Pesquisas têm documentado associações entre relacionamentos familiares - incluindo as relações maritais e entre pais e filhos - e problemas de ajustamento em crianças (Erel; Burman, 1995; Harold; Conger, 1997; Erath, et al, 2006). Alguns estudos demonstraram que o estabelecimento de uma relação conjugal insatisfatória pode ocasionar diversas conseqüências diretas e indiretas, tanto para os casais quanto para seus filhos (Gottman, 1993). Dentre os diversos prejuízos que as relações conjugais ruins parecem exercer sobre as crianças, estão os problemas de saúde física, as dificuldades 
acadêmicas, a depressão, a diminuição de habilidades sociais e os distúrbios de comportamento (Gottman, 1998).

Braz et al. (2005) demonstraram que o estabelecimento de bom relacionamento conjugal favorece a divisão das tarefas domésticas e das práticas de educação entre ambos os cônjuges, o que, por sua vez, promove desenvolvimento de sentimentos de segurança em seus filhos. A maioria dos cônjuges deste estudo acreditava que o seu relacionamento tinha influência, tanto direta quanto indireta, nas relações mantidas com a criança, assim como também percebiam o filho, como interferindo no relacionamento marital, corroborando os achados de outros autores (Belsky, 1984; Deal et al., 1999; Kreppner, 2000) sobre a influência do casal no filho. Além disso, Braz et al. (2005) observaram uma maior proporção de casais de classe média satisfeitos com seus relacionamentos maritais em comparação aos de classe mais baixa, indo ao encontro de resultados previamente publicados (Fletcher et al., 1999; Gottman, 1998).

Logo, a exposição ao conflito marital não possui um efeito uniforme. Seu impacto parece depender da combinação de vários eventos e da interação existente dentro da própria família, o que pode explicar, ao menos em parte, o porquê de algumas crianças expostas ao conflito apresentarem sintomas depressivos, terem reações agressivas ou sentimento de rejeição por um dos pais, enquanto outras crianças - principalmente aquelas que possuem bom relacionamento com seus pais - atribuem os conflitos a causas externas e temporárias, não apresentando problemas no seu desenvolvimento e na sua saúde (Fincham et al., 1994).

\section{Relacionamento marital em famílias com crianças com TDAH}

Nos últimos anos, alguns pesquisadores têm se dedicado a compreender fatores familiares potencialmente associados ao TDAH, modificando o prejuízo associado e/ou alterando o seu curso. Também tem sido alvo de investigação o impacto do TDAH no relacionamento conjugal dos pais de crianças portadoras.

Muitos estudos com amostras clínicas e epidemiológicas (Biederman et al., 1999; Biederman et al., 2006; DuPaul et al., 2001; Presentación et al., 2006; Scahill et al., 1999) revelaram níveis mais altos de estresse e ambiente familiar mais conflituoso em famílias de crianças com TDAH quando comparadas a controles (Biederman et al., 2006). Scahill et al. (1999), com o objetivo de identificar fatores psicossociais e clínicos relacionados ao TDAH, analisaram uma amostra comunitária de 449 crianças em região rural dos EUA, e concluíram que crianças do grupo com TDAH tinham níveis mais altos de disfunção familiar, dentre outros fatores relevantes, como história materna de tratamento psiquiátrico e pais com histórico de abuso de álcool. Além disso, os autores notaram que o aumento da gravidade dos sintomas relacionava-se a uma elevação dos níveis de prejuízos desses fatores, o que sugere que a adversidade psicossocial está associada com o aumento do grau de severidade do TDAH, apoiando o aspecto dimensional do transtorno.

Em relação especificamente ao relacionamento conjugal dos pais destas crianças, os achados se revelaram inconsistentes. Alguns estudos (Befera e Barkley, 1985; Presentación et al., 2006, Shelton et al., 1998) demonstraram que pais de crianças com TDAH, de diferentes idades e níveis de gravidade, relatavam uma menor satisfação marital e mais conflito do que pais de crianças sem TDAH. No Brasil, uma pesquisa da análise de contribuição de fatores de risco psicossociais para a ocorrência do transtorno, realizada por Vasconcelos et al. (2005), encontrou que as crianças expostas a brigas conjugais tinham um risco 11,66 vezes maior de ter o diagnóstico de TDAH.

Pais de crianças com TDAH tendem a se classificar como menos competentes na sua função como pais (Dewolfe et al., 2000) e a perceber sintomas de desatenção e comportamentos de desafio e oposição, menos controláveis e mais estáveis, tendendo a reagir mais negativamente a tais sintomas em comparação a grupos-controles (Johnston; Freeman, 1997). Talvez a diminuição da satisfação conjugal em pais de crianças com TDAH possa estar relacionada a esses fatores.

Em um recente estudo com amostra epidemiológica, Presentación et al. (2006) investigaram, por meio da análise da percepção dos pais de crianças com idade entre 7 e 10 anos e TDAH do subtipo combinado, uma série de aspectos da vida social, das dificuldades em lidar com a criança e do relacionamento marital. Os autores observaram uma diferença significativa em relação ao grupo-controle, demonstrando uma importante deterioração no funcionamento familiar e no relacionamento conjugal do grupo de pais das crianças com o transtorno do subtipo combinado, sendo o impacto ainda mais grave naquelas que também apresentavam transtorno de conduta. Este achado corrobora a idéia de que o tipo combinado do TDAH estaria mais fortemente associado a funcionamento familiar negativo do que os tipos com predomínio de desatenção ou predomínio de hiperatividade-impulsividade.

Os achados do estudo realizado por Counts et al. (2005) também sugeriram que famílias de crianças com o tipo combinado de TDAH apresentavam mais fatores de risco associados com adversidade familiar, como conflito marital, pais com histórico de transtornos psiquiátricos e eventos de vida estressantes, quando comparadas a famílias de crianças com o tipo predominantemente desatento e grupo-controle. Este estudo demonstrou que os sintomas de TDAH não estão associados às percepções das crianças acerca do conflito marital.

Já outras pesquisas não confirmam a associação entre ambiente familiar conflituoso e famílias com prole com o transtorno (Byrne et al., 1998; Cunningham et al., 1988). Em um estudo com amostra epidemiológica, Szatmari et al. (1989) demonstraram não haver mais relatos de problemas maritais entre pais de crianças portadores de TDAH, quando comparados a pais de controles normais. Barkley et al. (1990) também não encon- 
traram diferenças significativas entre famílias de portadores de TDAH e famílias de controles normais, de acordo com o auto-relato das mães, no que diz respeito à discórdia marital. Não foram encontrados índices aumentados de divórcios em pais de portadores de TDAH em diversos estudos (Barkley et al., 1991; Brown e Pacini, 1989; Faraone et al., 1991). De modo similar, Camparo et al. (1994) não encontraram maiores níveis de conflito marital utilizando questionários e entrevistas em uma amostra clínica de famílias de crianças com TDAH (idades entre 6 e 12 anos), quando comparadas a famílias de controles. No entanto, pais de portadores de TDAH censuravam mais seus filhos pelos problemas familiares.

Em relação à associação do conflito marital em pais ao subtipo combinado do transtorno nos filhos, existem estudos discordantes como os de Barkley et al. (1991) e Roselló et al. (2003). Nestas pesquisas, que abrangeram uma amostra de pais de crianças com TDAH combinado, a porcentagem de separação e divórcios não se revelou especialmente elevada, à semelhança do que foi observado em outros estudos (Brown e Pacini, 1989; Faraone et al., 1991), e os problemas maritais encontrados foram mais relacionados com uma convivência difícil, que afeta toda a família, e não apenas o casal.

Alguns estudos sugeriram que a inconsistência encontrada na relação entre funcionamento marital e prole com TDAH poderia ser explicada pela ocorrência da comorbidade com distúrbio desafiador e de oposição e/ou distúrbio de conduta. A relação entre discórdia marital e problemas infantis de conduta tem se mostrado consistente em numerosos estudos, especialmente no que se refere a amostras masculinas (Campbell, 1995; Dodge et al., 1994).

Um estudo de Barkley et al. (1992) encontrou pior ajustamento marital em pais de adolescentes com TDAH comórbido com DDO do que nos grupos somente com TDAH e controles. Johnston e Behrenz (1993) demonstraram que casais com crianças portadoras de TDAH que também apresentavam agressividade eram mais negativos quando discutiam entre si do que pais de crianças com TDAH sem agressividade ou do grupo-controle. Ainda nesta direção, Lindahl (1998) investigou, por meio de uma combinação de métodos de auto-relato e dados observacionais, múltiplos fatores familiares e parentais, incluindo o relacionamento marital. Para isso, avaliou quatro grupos de pais de crianças entre 7 e 11 anos: pais de crianças com TDAH, pais de crianças com DDO, pais de crianças com TDAH e DDO e pais de controles normais. Os resultados indicaram que pais das crianças com TDAH e DDO comórbido apresentavam maiores níveis de insatisfação marital do que o grupo apenas com TDAH. Cumpre ressaltar que os níveis de insatisfação marital de pais de portadores de DDO revelaram-se semelhantes aos encontrados em pais de crianças com os dois transtornos, sugerindo uma maior influência negativa do DDO no relacionamento marital.

Em contraste com estes estudos que relacionam a disfunção marital à presença da comorbidade, outras pesqui- sas não revelaram diferenças significativas no funcionamento marital em famílias de meninos com TDAH com ou sem comorbidade com problemas comportamentais (Barkley et al., 1991; Biederman et al., 1987; Prinz et al., 1983; Schacha e Wachsmuth, 1990).

Além da presença de comorbidades e da associação com o subtipo do transtorno, outros fatores devem ser considerados ao se tratar do relacionamento conjugal entre casais. Estudos têm evidenciado a importância de se considerar a saúde mental dos cônjuges. Conflito marital em homens e mulheres se associa a níveis mais altos de sintomas depressivos (O'Leary et al., 1994; Whiffen; Gotlib, 1989). Estes, por sua vez, poderiam agir como estressor generalizado sobre a família, induzindo a afetos negativos nos membros e nas habilidades de enfrentamento da criança (Downey e Coyne, 1990; Gelfand e Teti, 1990).

Uma pesquisa epidemiológica de Cunningham e Boyle (2002) sobre aspectos do funcionamento familiar e parental em famílias de crianças pré-escolares com TDAH revelou que as mães destas crianças apresentavam escores de depressão mais altos que as mães de crianças sem o transtorno. Kilic e Sener (2005) encontraram elevadas taxas de depressão materna e alcoolismo paterno em pais de crianças com TDAH comórbido com DDO. Lesesne et al. (2003) demonstraram que mães de crianças portadoras de TDAH apresentam maiores taxas de depressão e ansiedade, principalmente em mães solteiras e de baixo status socioeconômico (Whisman, 2001 apud Low e Stocker, 2005). Os achados de Low e Stocker (2005) sugerem que o humor depressivo em pais e a hostilidade marital em mães estão diretamente associados a problemas externalizantes nos fiIhos. Uma pesquisa realizada por Kashdan et al. (2004) teve como resultado uma associação negativa entre ansiedade nos pais e fatores como envolvimento parental, disciplina intrusiva e negativa e problemas sociais relacionados a sintomas opositivos e desafiadores nos filhos, no entanto não foi encontrada nenhuma relação específica com o TDAH.

Estudos epidemiológicos têm encontrado relatos de conflito marital em homens e mulheres em geral associados a níveis mais altos de sintomas depressivos (O'Leary et al., 1994; Whiffen e Gotlib, 1989). Aparentemente, o humor depressivo aparece freqüentemente associado à hostilidade marital e algumas pesquisas têm documentado uma ligação entre este tipo de conflito e problemas tanto externalizantes, incluindo o TDAH, quanto internalizantes em crianças (Cumming e Davies, 1994; Grych e Fincham, 1990).

A questão da comorbidade novamente se apresenta em um estudo realizado no Japão, por Satake et al. (2004), que demonstrou que a saúde mental de mães de crianças com TDAH comórbidos com DDO ou distúrbio de conduta era pior quando comparada com os grupos de mães com filhos apenas com TDAH e controles. 


\section{DISCUSSÃO}

Embora o relacionamento marital seja destacado na literatura como fator relevante para a compreensão dos problemas de ajustamento e do desenvolvimento infantil, o grau de dificuldade experimentado por pais de crianças portadoras de TDAH permanece incerto.

Apesar de diversos estudos documentarem uma associação significativa entre conflito marital e problemas de ajustamento infantil (Cumming e Davies, 2002), outras variáveis coexistentes e possíveis mediadores (como transtornos comórbidos) e características específicas de tais conflitos permanecem sendo importantes para a compreensão mais completa desta associação. Não parecem claramente estabelecidos os seguintes pontos: 1) se os problemas conjugais podem ser considerados secundários à presença de TDAH na prole; 2) se apenas casos de TDAH com comorbidade com transtornos disruptivos se associam a problemas conjugais; 3) se conflitos conjugais preexistentes interferem na manifestação dos sintomas de TDAH que, por sua vez, retro-alimenta os conflitos conjugais; 4) se a presença de TDAH (transtorno com elevada participação genética) em um dos cônjuges tem papel saliente no entendimento dos conflitos conjugais de modo independente da presença de TDAH na prole; e 5) se a presença de depressão ou ansiedade está relacionada ao impacto da presença de TDAH na prole.

\section{CONCLUSÕES}

Observa-se grande discordância de resultados nos estudos que avaliam a associação entre as relações familiares, conflitos conjugais e crianças portadoras de TDAH. Embora haja predomínio de resultados que sugerem a existência de dificuldades maiores nos relacionamentos familiares de crianças com TDAH, principalmente naquelas famílias em que as crianças também apresentam comorbidades com transtornos disruptivos, os estudos não apresentam concordância quanto a este aspecto.

O esclarecimento de questões importantes acerca dos mecanismos e processos de desenvolvimento que operam nesta relação é necessário, uma vez que fornecerá embasamento teórico para intervenções psicoterápicas. Estudos prospectivos seriam muito úteis para se determinar a direção da possível associação entre TDAH e discórdia marital. O impacto de tratamentos farmacológicos e/ou psicoterápicos do TDAH sobre os conflitos conjugais, bem como a influência da terapia dos conflitos conjugais sobre o TDAH merece ser estudada.

\section{REFERÊNCIAS}

American Psychiatric Association. Manual diagnóstico e estatístico dos transtornos mentais. 4a ed. Porto Alegre: Artes Médicas, 2000.

Andrade SA, Santos DN, Bastos AC, Pedromônico MRA, Almeida-Filho N, Barreto ML. Ambiente familiar e desenvolvimento cognitivo infantil: uma abordagem epidemiológica. Rev Saúde Pública 39(4): 606- 611, 2005.

Arnold EH, O'leary SG, Edwards GH. Father involvement and self-reported parenting of children with attention deficit-hyperactivity disorder. Journal of Consulting \& Clinical Psychology 65(2): 337-42, 1997.

Barkley R. Transtorno de déficit de atenção/ hiperatividade. Porto Alegre: Ed. Artmed, 2002.

Barkley RA, Anastopoulos AD, Guevremont DC, Fletcher KE. Adolescents with attention deficit hyperactivity disorder: Mother adolescent interactions, family beliefs and conflicts, and maternal psychopathology. Journal of Abnormal Child Psychology 20: 263-288, 1992.

Barkley RA, Dupaul GJ, Mcmurray MB. Comprehensive evaluation of attention deficit disorder with and without hyperactivity as defined by research criteria. Journal of Consulting and Clinical Psychology 58: 775-789, 1990.

Barkley RA, Fischer M, Edelbrock C, Smallish L. The adolescent outcome of hyperactive children diagnosed by research criteria. III. Mother child interactions, family conflicts and maternal psycopathology. Journal of Child Psychology and Psychiatry 32: 233-255, 1991.

Befera MS, Barkley RA. Hyperactive and normal girls and boys: mother child interaction, parent psychiatric status and child psycho pathology. Journal of Child Psychology and Psychiatry 26: 439-452, 1985.

Belsky J. The determinants of parenting: a process model. Child Development 17: 3-23, 1984.

Berger A, Posner MI. Pathologies of brain attentional networks. Neuroscience and Biobehavioral Reviews 24: 3-5, 2000

Biederman J, Faraone SV, Mick E, Williamson S, Wilens TE, Spencer TH, Weber W, Jetton J, Kraus I, Pert J, Zallen B. Clinical correlates of ADHD in females: findings from a large group of girls ascertained from pediatric and psychiatric referral sources. Journal of the American Academy of Child and Adolescent Psychiatric 38: 966-975, 1999.

Biederman J, Monuteaux MC, Mick E, Spencer T, Wilens TE, Klein KL, Price JE, Faraone SV. Psychopathology in females with Attention-Deficit / Hyperactivity Disorder: a controlled, five-year prospective study. Biol Psychiatry 60:1098-1105, 2006.

Biederman J, Munir K, Knee D. Conduct nad oppositional disorder in clinically referred children with attention deficit disorder: A controlled family study. Journal of the American Academy of Child and Adolescent Psychiatry 26:724-727, 1987.

Bradley RH, Corwyn RF. Socioeconomic status and child development. Annual Review Psychology 53:371-399, 2002.

Braz MP, Dessen MA, Silva NLP. Relações conjugais e parentais: uma comparação entre famílias de classes sociais baixa e média. Psicologia Reflexão e Crítica 18 (2): 151-161, 2005.

Bronfenbrenner U, Ceci SJ. Nature-nurture reconceptualized in developmental perspective: a bioecological model. Psychol Rev 101 (4): 568-586, 1994.

Brown RT, Pacini JN. Perceived family functioning, marital status, and depression in parents of boys with attention deficit disorder. Journal of Learning Disabilities 22: 581-587, 1989.

Byrne JM, De Wolfe NA, Bawden HN. Assessment of attention déficit hyperactivity disorder in preschoolers. Child Neuropsychology 4: 49-66, 1998.

Camparo L, Christensen A, Buhrmester D, Hinshaw S. System functioning in families with ADHD and non-ADHD sons. Personal Relationships 1: 301-308, 1994.

Campbell SB. Behavior problems in preschool children: A review of recent research. Journal of Child Psychology and Psychiatry 36: 113-149, 1995.

Carlson EA, Jacobvitz D, Sroufe LA. A developmental investigation of inattentiveness and hyperactivity. Child Development 66: 37-54, 1995

Counts CA, Nigg JT, Stawicki JA, Rappley MD, Von Eye A. Family adversity in DSM-IV ADHD combined and inattentive subtypes and associated disruptive behavior problems. Journal of the American Academy of Child \& Adolescent Psychiatry. 44(7):690-698, 2005.

Cummings EM, Davies PT. Effects of marital conflict on children: recent advances and emerging themes in process-oriented research. Journal of Child Psychology and Psychiatry and Allied Disciplines 43: 31-63, 2002. 
Cummings EM, Davies PT. Maternal depression and child development. Journal of Child Psychology and Psychiatry and Allied Disciplines 35: 73-112, 1994.

Cunningham CE, Benness BB, Siegel LS. Family functioning, time allocation, and parental depression in the families of normal and ADHD children. Journal of Clinical Child Psychology 17: 169-177, 1988

Cunningham CE, Boyle MH. Preschoolers at risk for attention-deficit hyperactivity disorder and oppositional defiant disorder: family, parenting, and behavioral correlates. Journal of Abnormal Child Psychology 30 (6): 555-569, 2002.

Deal JE, Hagan MS, Bass B, Hetherington EM, Clingempeel G. Marital interaction in dyadic and triadic contexts: continuities and discontinuities. Family Process 38: 105-115, 1999.

DeWolfe N, Byrne JM, Bawden HN. ADHD in preschool children: parent-rated psychosocial correlates. Developmental Medicine \& Child Neurology. 42(12): 825-830, 2000.

Dodge KA, Pettit GS, Bates JE. Socialization mediators of the relation between socioeconomic status and child conduct problems. Child Develpoment 65: 649-665, 1994.

Downey G, Coyne J. Children of depressed parents: An integrative review. Psychological Bulletin 108: 50-76, 1990.

DuPaul GJ, Mccgoey KE, Eckert TL, Vanbrakle J. Preschool children with Attention-Deficit Hyperactivity Disorder : impairments in behavioral, social and school functioning. Journal of the American Academy of Child and Adolescent Psychiatric 40: 508-515, 2001.

Erath SA, Bierman KL, Conduct Problems Prevention Research Group. Aggressive marital conflict, maternal harsh punishment, and child aggressive-disruptive behavior: evidence for direct and mediated relations. Journal of Family Psychology. 20(2):217-26, 2006.

Erel 0, Burman B. Interrelatedness of marital relations and parent-child relations: a metaanalytic review. Psychological Bulletin 118: 108-132, 1995.

Eriksson T. Antiandrogenic treatment for obsessive-compulsive disorder. Am J Psychiatry, 157(3):483, 2000.

Faraone SV, Biederman J, Keenan K, Tsuang MT. Separation of DSM-III attention deficit disorder and conduct disorder: Evidence from a family-genetic study of American child psychiatric patients. Psychological Medicine 21: 109-121, 1991.

Faraone SV, Biederman J. Neurobiology of attention deficit hyperactivity disorder. Biological Psychiatry 44: 951-958, 1998.

Fauber R, Long N. Children in context: the role of the family in child psychotherapy. Journal of Consulting and Clinical Psychology 59: 813-820, 1991

Fincham FD, Grych JH, Osborne LN. Does marital conflict cause child maladjustment? Directions and challenges for longitudinal research. Journal of Family Psychology 8(2): 128-140, 1994.

Fletcher GJO, Simpson JA, Thomas G, Giles L. Ideals in intimate relationships. Journal of Personality ans Social Psychology 76: 72-89, 1999.

Fontenelle LF, Mendlowicz MV, Marques C, Versiani M. Early- and late-onset obsessive-compulsive disorder in adult patients: an exploratory clinical and therapeutic study. J Psychiatr Res, 37(2):127-33, 2003

Gelfand DM, Teti DM. The effects of maternal depression on children. Clinical Psychology Review 10: 329-353, 1990

Gottman JM. Psychology and the study of marital processes. Annual Review of Psychology 49: 169-197, 1998.

Gottman JM. The roles of conflict engagement, escalation, and avoidance in marital interaction: a longitudinal view of five types of couples. Journal of Consulting and Clinical Psychology 61: 6-15, 1993

Grych J, Fincham F. Marital conflict and children's adjustment: A cognitive-contextual framework. Psychological Bulletin 108: 267-290, 1990.

Grych JH, Fincham FD. Children's appraisals of marital conflict: initial investigations of the cognitive-contextual framework. Child Development 64: 215-230, 1993.

Grych JH, Seid M, Fincham FD. Assessing marital conflict from the child's perspective: the children's perceptions of interparental conflict scale. Child Development 63: 558-572, 1992.

Harold GT, Conger RD. Marital conflict and adolescent distress: the role of adolescent awareness. Child Develpoment 68: 243-260, 1997.

Harpin VA. The effect of ADHD on the life of an individual, their family, and community from preschool to adult life. Arch Dis Child 90(1): 2-7, 2005

Hechtman L. Families of children with attention deficit hyperactivity disorder: a review. Canadian Journal of Psychiatry - Revue Canadienne de Psychiatrie 41(6): 350-360, 1996.

Johnston C, Behrenz K. Childrearing discussions in families of nonproblem children and ADHD children with higher and lower levels of aggressive-defiant behavior. Canadian Journal of School Psychology 9: 53-65, 1993.
Johnston C, Freeman W. Attributions for child behavior in parents of children without behavior disorders and children with attention deficit-hyperactivity disorder. Journal of Consulting \& Clinical Psychology 65(4):636-645, 1997.

Johnston C, Mash EJ. Families of children with attention-deficit/hyperactivity disorder: review and recommendations. Clinical Child and Family Psychology Review 4(3): 183-207, 2001.

Kashdan TB, Jacob RG, Pelham WE, Lang AR, Hoza B, Blumenthal JD, Gnagy EM. Depression and anxiety in parents of children with ADHD and varying levels of oppositional defiant behaviors: modeling relationships with family functioning. Journal of Clinical Child \& Adolescent Psychology 33(1): 169-81, 2004.

Kilic B, Sener S. Family functionig e psychosocial characterisitcs in children with attention deficit hyperactivity disorder with comorbid oppositional defiant disorder or conduct disorder. Turk Psikiyatri Dergisi 16(1): 21-28, 2005.

Kreppner $\mathrm{K}$. The child and the family: interdependence in developmental pathways. Psicologia: Teoria e Pesquisa 16: 11-22, 2000.

Lesesne CA, Visser SN, White CP. Attention-deficit disorder in school-aged children: Association with maternal mental health and use of health care resources. Pediatrics 111(5): 1232-1237, 2003.

Lindahl KM. Family process variables and children's disruptive behavior problems. Journal of Family Psychology 12(3): 420-436, 1998.

Low SB, Stocker C. Family functioning and children's adjustment: Associations among parent's depressed mood, marital hostility, parent-child hostility and children's adjustment. Journal of family psychology 3: 394-403, 2005.

Miranda A, García R, Presentación MJ. Factores moduladores de la eficácia de uma intervención psicosocial em niños com transtorno por déficit de atención com hiperctividad. Revista de Neurologia 34(1): 91-97, 2002.

O'leary KD, Christian JL, Mendall N. A closer look at the link between marital discord and depression. Journal of Social and Clinical Psychology 13: 33-41, 1994.

Presentacion MJ, García R, Miranda A, Siegenthaler R, Jara P. Familial impact of children with the combined subtype off attention deficit hyperactivity disorder: the effects of associated behavioural disorders. Revista de Neurologia 42 (3): 137-143, 2006.

Prinz RJ, Myers D, Holden EW, Tarnowski J, Roberts WA. Marital disturbance and child problems: A cautionary note regarding hyperactive children. Journal of Abnormal Child Psychology 11: 393-399, 1983.

Rohde LA et al. Transtorno de déficit de atenção/hiperatividade na infância e na adolescência: considerações clínicas e terapêuticas. Revista de Psiquiatria Clínica 31(3): 124-131, 2004.

Rosario-Campos MC, Leckman JF, Mercadante MT, Shavitt RG, Prado HS, Sada P, Zamignani D, Miguel EC. Adults with early-onset obsessive-compulsive disorder. Am J Psychiatry, 158(11):1899-903, 2001.

Roselló B, García R, Tárraga J, Mulas F. El papel de los padres en el desarrollo y aprendizaje de los niños com trastorno por déficit de atención com hipercatividad. Rev Neurol 36: 79-84, 2003

Satake H, Yamashita H, Yoshida K. The family psychosocial characteristics of children with attention-deficit hyperactivity disorder with or without oppositional or conduct problems in Japan. Child Psychiatry \& Human Development. 34 (3): 219-235, 2004.

Scahill L, Schwab-Stone M, Merikangas KR, Leckman JR, Zhang H, Kasl S. Psychosocial and clinical correlates of ADHD in a community sample of school age children. Journal of the American Academy of Child and Adolescent Psychiatry 38: 976-984, 1999.

Schachar R, Wachsmuth R. Hyperactivity and parental psychopathology. Journal of Child Psychology and Psychiatry 31: 381-392, 1990.

Shelton TL, Barkley RA, Crosswait C, Moorehouse M, Flecther K, Barret S, Jenkins L, Metevia L. Psychiatric and psychological morbidity as a function of adaptive disability in preschool children with agressive and hyperactive impulsive inattentive behavior. Journal of Abnormal Child Psychology 26: 475-494, 1998.

Souza RM. A criança na família em transformação: um pouco de reflexão e um convite à investigação. Psic Rev 5: 33-51, 1997.

Szatmari P, Boyle MH, Offord, DR. ADHD and conduct disorder: Degree of diagnostic overlap and differences among correlates. Journal of American Academy of Child and Adolescent Psychiatry 28: 865-872, 1989.

Vasconcelos MM, Malheiros AFA, Werner Jr J, Brito AR, Barbosa JB, Santos IS, Lima DF. Contribuição dos fatores de risco psicossociais para o transtorno de déficit de atenção/hiperatividade. Arquivos de Neuro-Psiquiatria 63(1): 68-74, 2005.

Whiffen VE, Gotlib IH. Stress and coping in maritaly distressed and nondistressed couples. Journal of Social and Personal Relationships 6: 327-344, 1989.

Wolfe DA. Child abusive parents: an empirical review and analysis. Psychological Bulletin 97: 462-487, 1985. 